This is the post peer-review accepted manuscript of:

Schultze-Krumbholz, A., Göbel, K., Scheithauer, H., Brighi, A., Guarini, A., Tsorbatzoudis, H., Barkoukis, V., Pyżalski, J., Plichta, P., Del Rey, R., Casas, J. A., Thompson, F., \& Smith, P. K. (2015). A Comparison of Classification Approaches for Cyberbullying and Traditional Bullying Using Data From Six European Countries. Journal of School Violence, 14(1), 4765. https://doi.org/10.1080/15388220.2014.961067

The published version is available online at: https://doi.org/10.1080/15388220.2014.961067

Rights / License:

The terms and conditions for the reuse of this version of the manuscript are specified in the publishing policy. For all terms of use and more information see the publisher's website. 


\title{
A Comparison of Classification Approaches for Cyberbullying and Traditional Bullying Using Data from Six European Countries
}

\author{
ANJA SCHULTZE-KRUMBHOLZ \\ Department of Educational Science and Psychology, Freie Universität Berlin, Germany \\ KRISTIN GÖBEL \\ Department of Educational Science and Psychology, Freie Universität Berlin, Germany \\ HERBERT SCHEITHAUER
}

Department of Educational Science and Psychology, Freie Universität Berlin, Germany ANTONELLA BRIGHI

Department of Education Sciences, University of Bologna, Italy

ANNALISA GUARINI

Department of Psychology, University of Bologna, Italy

HARALAMBOS TSORBATZOUDIS

School of Physical Education and Sports Science, Aristotle University of Thessaloniki, Greece

\section{VASSILIS BARKOUKIS}

School of Physical Education and Sports Science, Aristotle University of Thessaloniki,

Greece

\section{JACEK PYŻALSKI}

Faculty of Educational Studies, Adam Mickiewicz University in Poznań, Poland PIOTR PLICHTA

Faculty of Historical and Pedagogical Sciences, University of Wrocław, Poland ROSARIO DEL REY

Department of Developmental and Educational Psychology, University of Seville, Spain 


\author{
JOSÉ A. CASAS \\ Department of Education, Catholic University of San Antonio (Murcia), Spain \\ FRAN THOMPSON \\ Department of Psychology, Goldsmiths, University of London, U.K. \\ PETER K. SMITH \\ Department of Psychology, Goldsmiths, University of London, U.K.
}

Date submitted: July 25, 2014; date accepted: August 30, 2014.

Address correspondence to Freie Universitaet Berlin, Department of Educational Science and Psychology, Habelschwerdter Allee 45, Berlin 14195, Germany. E-mail: anja.schultze-krumbholz@fu-berlin.de 


\begin{abstract}
In recently published studies on cyberbullying, students are frequently categorized into distinct (cyber-)bully, and (cyber-)victim clusters based on theoretical assumptions and arbitrary cutoff scores adapted from traditional bullying research. The present study identified involvement classes empirically using latent class analysis (LCA), to compare the classification of cyber- and traditional bullying and to compare LCA and the conventional approach. Participants were 6,260 students $(M=14.8$ years, $S D=1.6 ; 49.1 \%$ male $)$ from six European countries. LCA resulted in three classes for cyberbullying and four classes for traditional bullying. Cyber- and traditional bullying differed from each other, as did LCA and the conventional approach. Country, age and gender differences were found. Implications for the field of traditional and cyberbullying research are discussed.
\end{abstract}

KEYWORDS cybervictimization, cyberbullying, bullying, latent class analysis, crossnational data, classification 


\section{A comparison of classification approaches for cyberbullying and traditional bullying using data from six European countries}

\section{Introduction}

The body of literature on cyberbullying has increased very rapidly over the last decade. Cyberbullying is conceived as a form of aggression using electronic or digital media for repeated hostile communications to intentionally harm others (cf. Tokunaga, 2010). Prevalence rates range from $6 \%$ to $72 \%$ for victimization and from $4 \%$ to $36 \%$ for perpetration (cf. Suzuki, Asaga, Sourander, Hoven, \& Mandell, 2012). Reviews of international studies found mean prevalence rates of $24 \%$ for victimization and $16-18 \%$ for perpetration (Patchin \& Hinduja, 2012; Suzuki et al., 2012). These or similar (e.g., national) rates often provide the basis for demands for action, intervention, and prevention approaches.

Cyberbullying research developed from traditional bullying research, which already provided decades of findings when cyberbullying research first emerged in the early 2000s (cf. Smith, 2010). Thus, research questions were often inspired by what was already known about traditional bullying which is commonly defined as repeated, intentional aggressive behavior by a group or individual against a victim who cannot easily defend him- or herself (Olweus, 1993). Recently, there has been debate about whether cyberbullying is just a subtype of traditional bullying, and distinctive features have been highlighted repeatedly (for specific features see e.g., Li, Smith, and Cross [2012]). Besides, studies have shown that cyberbullying instruments do not show the same factor structure as instruments for traditional bullying. A measure specifically designed for the cyber context by Law, Shapka, Hymel, Olson, and Waterhouse (2012) found that the traditional bullying items loaded on two distinct factors (one for bullying and one for victimization), while all the cyberbullying and cybervictimization items loaded on one factor, a single "cyberbullying/victimization" factor. Further analyses revealed that this factor could be subdivided into "aggressive messaging" 
and "embarrassing pictures," but that perpetration and victimization were still inextricably linked. Menesini, Nocentini, and Calussi (2011) obtained similar results and found cyberbullying (including cybervictimization) to be unidimensional and continuous regarding severity. Nonetheless, the conventional classification approach for traditional bullying has often been transferred to cyberbullying resulting in the common classes of "victim," "bully," "bully-victim," and "noninvolved." In this approach, a cutoff score is set either if at least one item fulfills a predefined frequency (e.g., "2-3 times a month") or when a total score exceeds a group-based standardized magnitude such as one standard deviation above a school class's mean (cf. Nylund, Bellmore, Nishina, \& Graham, 2007).

There are no previous studies using latent class analysis (LCA) to examine subtypes of cyberbullying involvement and only a few on peer harassment and peer victimization in general. LCA is a person-centered and model-based method assigning individuals to similar patterns across a set of responses while keeping the distinction between groups high. Wang, Iannotti, Luk, and Nansel (2010) included cybervictimization as one possible form of peer victimization along with forms of traditional peer victimization to identify subtypes of peer victimization in general and found the classes "nonvictims," "all-type victims" (including cybervictimization), and "verbal/relational victims" (not including cybervictimization). However, victimization through cyberbullying was assessed using two global items, which were then collapsed into one dichotomous indicator. Another study including a global cyberbullying item as just one indicator of more comprehensive peer victimization found the classes "low victimization/normative," "high verbal, physical and relational victimization" (including cybervictimization), "verbal and physical" (not including cybervictimization), and "verbal and relational" victimization (not including cybervictimization) for middle school students (Bradshaw, Waasdorp, \& O’Brennan, 2013). The reported studies focused on victimization and therefore did not examine the validity of the common four conventional 
involvement groups. Regarding these, results from the few existing studies integrating both victimization and perpetration of general peer harassment are inconsistent and are only able to partly replicate the four conventional classes (e.g., Lovegrove, Henry, \& Slater, 2012). Others found either a five-class solution, which differentiated bully-victims further (Giang \& Graham, 2008), or a three-class solution with no exclusive bully class (Williford, Brisson, Bender, Jenson, \& Forrest-Bank, 2011). So the ecological validity of the theoretical conventional classes is still unclear and the transferability of this predominant classification approach to cyberbullying data also lacks empirical support.

Apart from examining classes of cyberbullying involvement, the present study included the demographic variables age, gender, and country. Previous studies have shown cyberbullying to peak in middle school (e.g., Ortega, Elipe, Mora-Merchán, Calmaestra, \& Vega, 2009) and then to constantly increase through high school (Patchin \& Hinduja, 2012) or to just constantly increase across grade (Görzig \& Ólafsson, 2013; Wolak, Mitchell, \& Finkelhor, 2007). Results on gender differences are mixed, but a review of international empirical studies showed girls more likely to be victims and boys more likely to be perpetrators (Patchin \& Hinduja, 2012). Country differences have rarely been reported because very few cross-national studies have been conducted so far. Ortega and colleagues (2012) as well as Genta et al. (2012) reported differences in the involvement classes between Spain, Italy, and the United Kingdom (using the same study sample). Specifically, Genta et al. (2012) included 5,862 12- to 16-year-olds from Spain, Italy, and the UK, which were about evenly distributed across gender and year groups. They found Italian adolescents to be victims of cell phone bullying significantly more often than Spanish youths and girls more likely to be victims than boys. In contrast, they found more boys to perpetrate bullying by both cell phone and Internet, Italian participants to do so more than the Spanish adolescents, and these more than the UK students. Potential between-country gender differences were not 
reported. Further, the authors did not find age differences between Grades 8, 10, and 12. Lobe, Livingstone, Ólafsson, and Vodeb (2011) found country differences in victimization rates across 25 European countries in the EU Kids Online study, which used a stratified sample of 1,000 9- to 16 year-olds from a more comprehensive sample of over 25,000 children. The rates of six of the 25 countries are of special interest because they are also represented in the present study: $8 \%$ of UK participants reported being cybervictims, which is above the average of $6 \%$ across all 25 countries, while Poland (6\%), Germany (5\%), Spain (4\%), Greece (4\%), and Italy (2\%) showed average or well below average rates of cyberbullying victimization. Among these six European countries, gender of the participant was a significant predictor only in Spain, where girls were more likely to be victims of cyberbullying. The authors found no age differences.

\section{The Current Study}

In this article we examined whether the reported previous arbitrary classifications can be replicated empirically and whether there are differences in classification groups between cyberbullying and traditional bullying. The research objectives were therefore (a) to identify classes of cyberbullying involvement with empirical methods (more specifically with LCA), (b) to replicate the conventional classes of traditional bullying, (c) to compare extracted classes between cyber- and traditional bullying, (d) to compare the LCA and the conventional approach, and (e) to investigate the influence of the demographic variables gender, age and country on class membership.

Based on the findings from general peer harassment literature, we expected four classes for traditional bullying: (a) noninvolved students; (b) victims who are solely targets of bullying incidents; (c) bullies who are solely perpetrators of bullying incidents; and (d) bullyvictims who experience, but also perpetrate bullying. Following the arguments of some researchers who claim cyberbullying to be a subtype or an extension of traditional bullying 
(e.g., Olweus, 2012) we would expect these same classes to also emerge for cyberbullying. However, as mentioned before, the structure of cyberbullying behavior may differ. Therefore, the examination of cyberbullying classes is exploratory and we do not have specific hypotheses regarding the number and meaning of classes.

\section{Method}

\section{Procedure}

The present data were collected as part of the European Cyberbullying Intervention Project (for more information see also www.bullyingandcyber.net/en/ecip/project). Only cross-sectional data from the first measurement wave (collected between January and March 2011) before interventions were implemented on the national level were used for the present analyses. In line with national regulations, passive or active parents' consent as well as active students' consent was obtained before data collections, which were conducted by trained test instructors using paper questionnaires during regular school lessons. Prior to questionnaire administration students were informed that the questionnaire was anonymous, the participation in the study was voluntary and that they could withdraw at any time without any foreseeable consequences.

\section{Participants}

In total, 6,328 students from six European countries took part in the present data collection wave. Sixty-eight students $(1.1 \%)$ were excluded from the sample due to missing values on all items of at least one of the subscales, because these could not be estimated by full information maximum likelihood. This left a final sample of $n=6,260$ participants for the subsequent analyses. Participants were distributed across the different countries as follows: $16.0 \%(n=1,002)$ from Poland, $14.2 \%(n=892)$ from Spain, $27.1 \%(n=1,697)$ from Italy, $12.3 \%(n=771)$ from the United Kingdom, $14.3 \%(n=895)$ from Germany, and $16.0 \%(n=1,003)$ from Greece. Forty-nine percent of participants were male, $49.9 \%$ were 
female and $1.1 \%$ did not indicate their gender. The students' age ranged from 11 to 23 years $(M=14.8, S D=1.6)$. All students attended the national equivalent of high school, secondary school, or vocational school. The specific sample characteristics for each country can be found in Table 1.

[TABLE 1]

\section{Measures}

Apart from demographic background variables such as gender and age, cyberbullying and cybervictimization as well as traditional bullying and traditional victimization were assessed across all countries using the European Cyberbullying Intervention Project Questionnaire (ECIPQ; Brighi et al., 2012). This questionnaire used 12 items each to measure cyberbullying and cybervictimization. Due to ambiguity one item was deleted from each of these scales leaving 11 items each to measure perpetration of and victimization through cyberbullying. Students were asked to answer on a five-point scale $(1=$ no to $5=y e s$, more than once a week) whether they had experienced or taken part in any of the listed behaviors during the previous two months either online or through cell phones (see Table 1).

Traditional bullying and victimization was also assessed using the ECIPQ, which operationalized each of these scales through seven items (see Table 2). Corresponding to cyberbullying, students were again asked to answer on a five-point scale $(1=$ no to $5=y e s$, more than once a week) whether they had experienced or taken part in any of the listed behaviors during the previous two months.

For the present analyses, answers were trichotomized into no/not at all (coded as 0), only once or twice (coded as 1), and at least 2-3 times a month (coded as 2 in the subsequent LCA). The rationale for this approach was to provide results comparable to as large a number of studies as possible as the cutoff scores for classification across studies vary between only once or twice and at least 2-3 times a month. Also, it is still under debate, whether some 
behaviors can already be understood as cyberbullying although they only happen once (e.g., Langos, 2012).

[TABLE 2]

\section{Data Analysis Plan}

A Latent Class Analysis (LCA) was conducted using Mplus 7.1 statistical package (Muthén \& Muthén, 1998-2012) to identity patterns of victimization and bullying behavior.

The three-step approach was applied as the model included covariates. This stepwise approach starts with the model building process using a set of items, then it assigns individuals to the latent classes and finally estimates a multinomial logistic regression model (for more information see Vermunt, 2010).

In a series of analyses, successive LCA models with ascending number of classes were examined and then model selection was determined by evaluating underlying statistical evidence in cooperation with theoretical assumptions (Nylund et al., 2007). Subsequently, the appropriate number of latent classes was based on statistical criteria, which included loglikelihood value, Akaike information criterion (AIC), Bayesian information criterion (BIC), the sample size-adjusted Bayesian criterion (aBIC), and Bootstrap Likelihood Ratio Test (BLRT). Furthermore, the entropy and posterior probabilities for each model were examined. For models with similar levels of goodness of fit, the conceptual evidence and class interpretability were considered and the simpler model was chosen (Nylund et al., 2007; Waasdorp \& Bradshaw, 2011). LCA was run separately for cyberbullying and traditional bullying to determine the number of latent classes for each of those concepts. This approach was chosen because a comparison between the structures of the two phenomena was of interest while conclusions about the co-occurrence of traditional and cyberbullying were not an objective of the present study. Another reason was the model fit: when including all items of all four constructs (cyberbullying perpetration, cyberbullying victimization, traditional 
bullying perpetration, and traditional bullying victimization) the statistics program was not able to replicate the model sufficiently often indicating a lack of stability and too many parameters. Nevertheless, an exploratory factor analysis with all items has shown the constructs to be distinct except for two out of 36 items. After the best-fitted model was found, demographic differences between latent classes were examined using multinomial logistic regression. Specifically, we investigated whether country, age, or gender were associated with class membership.

For all analyses, a sandwich estimator was utilized to adjust for standard errors to account for the nesting of students within schools. Cases with missing at random (MAR) on some bullying items were included through estimation by full information maximum likelihood. Missing data for cyberbullying ranged from a low of $1.7 \%$ for victims of insults (for traditional bullying $1.7 \%$ for victims of physical bullying) to a high $3.3 \%$ for Photoshop perpetrators (for traditional bullying $4.8 \%$ for perpetrator of spreading rumors)

\section{Results}

The results are presented in three parts. First, the LCA results are provided, highlighting the structure and the number of classes for each bullying concept. Second, latent classes for cyberbullying are compared to the conventional method of assigning individuals. Finally, covariates (i.e., gender, age, country) are explored and tested for differences between latent classes.

\section{Model Selection and Number of Classes}

Table 3 provides details on fit information (i.e., AIC, BIC, aBIC, log-likelihood values) with an increasing number of classes for cyber- and traditional bullying. Inspection of the fit indices suggests a three-class model for cyberbullying and a four-class model for traditional bullying best fit the data as fit indices began to level off after the optional classes. The entropy of the three-class cyberbullying and the four-class traditional bullying model was 
.87 and .81 , respectively. Average posterior probabilities for the membership in each latent class were high (> .85). The Bootstrap Likelihood Ratio Test provided a significant $p$-value indicating a good fit. In addition, interpretation of classes was demonstrated to be meaningful and congruent with theoretical deliberations.

\section{[TABLE 3]}

The item probability distribution for each latent class of cyberbullying is shown in Figure 1. For cyberbullying, noninvolved individuals comprised $70.1 \%$ of the sample (Figure 1a). Further, two more classes were extracted (see Figures 1b and 1c): Bully-victims were present with $26.1 \%$ and showed a high probability of endorsing the verbal cybervictimization and cyberbullying items and relational cyberbullying items. About $4 \%$ of participants belonged to the perpetrator with mild victimization class. Participants endorsed high levels of cyberbullying, specifically verbal attacks, threats, stealing, and altering personal information. Cybervictimization was only mildly pronounced compared to cyberbullying.

For traditional bullying, noninvolved individuals, the biggest class of participants, comprised $43 \%$. Thirty-four percent of participants belonged to a mild bully-victim class. Participants in this class endorsed high levels of verbal victimization (e.g., insults, speak ill, rumors) and relational bullying (e.g., exclusion). Members of the third class for traditional bullying $(12.3 \%)$ had a relatively high probability of endorsing bullying items. This mainly perpetrator class showed high levels of physical bullying, verbal, and relational bullying. Perpetrators experienced comparatively lower levels of victimization. Eleven percent of participants showed bully-victim behavior with a focus on victimization. Members of this class had high probabilities of endorsing verbal, relational, and physical victimization items.

[FIGURES 1a, b, c]

\section{Comparison of Classification Approaches}

In the next step, the results of the typical classification method was compared to the 
LCA models by assigning students to one of four groups using the raw score. Students with a minimum of a one-time incident for any (cyber-)victimization item were labelled as victim, students with a minimum of a one-time incident for any (cyber-)bullying item were labelled as bully, students with a minimum of a one-time incident for any victimization and any bullying item were labelled as bully-victim and students with no incidents were labeled as noninvolved.

Students labeled as noninvolved were comprised of $41.0 \%$ of participants. The bullyvictim and the victim group was comprised of $24.2 \%$ and $26.8 \%$, respectively, and the bullies group was the smallest with $8.1 \%$.

Finally, latent classes and the conventional groups were examined for overlapping classifications. Table 4 shows the percentage of overlap between classifications for cyberbullying. Of those classified as bully-victims by LCA, $67.2 \%$ were also classified as such by the conventional approach. However, the other $32.8 \%$ of LCA-classified bullyvictims were categorized as victims and bullies by the conventional method. About $90 \%$ of perpetrators with mild victimization classified by the LCA were bully-victim with the conventional approach. In turn, none of the students classified as noninvolved by the conventional approach was classified otherwise by the LCA. Looking at the classes of the conventional approach, large proportions of involved students were classified as noninvolved by the LCA (75.0\% of conventional victims, $17.3 \%$ of conventional bully-victims, and $77.6 \%$ of conventional bullies).

[TABLE 4]

\section{Relation Between Class Membership and Demographics}

Gender, age and country were included as covariates to investigate the presence of differences among LCA classes.

We examined if boys or girls were more likely to be in a certain cyberbullying class. 
The noninvolved class was used as the reference group. The analysis of cyberbullying indicates that there were significant gender differences between the noninvolved and the other two classes. The odds of belonging to the bully-victim class compared to the noninvolved class was lower for boys $(\mathrm{OR}=0.47)$, but for belonging to the perpetrator class compared to the noninvolved class was higher for boys compared to girls $(\mathrm{OR}=1.19)$.

An investigation of age differences indicated that compared to the noninvolved classes, students in all other classes were significantly older.

Differences in country of data origin were visible. The between-country differences for cyberbullying were as follows: In Greece, the odds of belonging to the perpetrator with mild victimization class compared to the noninvolved class were higher compared to all other countries except Poland. Students from Germany were significantly less likely to belong to the perpetrator with mild victimization class compared to the noninvolved class in comparison to all other countries ( $\mathrm{OR}=0.26$ in reference to Greece). In Italy, the odds of belonging to the bully-victim class compared to the noninvolved class were higher compared to all other countries and especially the $\mathrm{UK}(\mathrm{OR}=0.36)$.

\section{Discussion}

To date, the present study is the first to conduct a latent class analysis on cyberbullying and cybervictimization data with multiple behavioral items. The goals were to apply the LCA method to classify students into classes based on their endorsement in an array of cyberbullying behaviors. Additionally, the results of the LCA are used to illustrate an alternative method to classify individuals into groups. The specific research objectives were to (a) identify classes of cyberbullying involvement using empirical data and statistical methods, (b) to examine whether the conventional theoretical classes of traditional bullying involvement can be empirically replicated, (c) to compare the LCA-based classifications of cyber- and traditional bullying, and (d) to compare the LCA-based classifications with the 
conventional classification. To this end, European data from six different countries was used and the association between class membership and the demographic covariates gender, age and country of data origin were examined.

\section{Classification of Cyberbullying Involvement}

Contrary to previous beliefs that cyberbullying is a subtype of traditional bullying (Olweus, 2012) and in line with studies showing structural differences to traditional bullying (Law et al., 2012; Menesini et al., 2011) we did not find a four-class solution with bullies, victims, bully-victims, and noninvolved individuals. Instead, a model with three classes noninvolved, bully-victims, and perpetrators with mild victimization exhibited the

This lack of an exclusive victim class was surprising, but might be explained with specific characteristics of the cyber context. For example, the online disinhibition effect which describes the phenomena of people sharing more information online or acting out more intensely than they would in real life (Suler, 2004) is facilitated by anonymity, invisibility and status equalization, among others. Possibly, victimized individuals lash back at their aggressors more easily than in real life where there is an actual status and strength difference between the involved parties. The lack of a clear "victim" class is further in line with numerous studies, which found substantial overlap between perpetration and victimization in cyberbullying (c.f. Kowalski, Guimetti, Schroeder, \& Lattanner, 2014). At the same time, the perpetrator with mild victimization class indicates that perpetrators are not free of victimization experiences themselves as has been found also for traditional peer harassment (Giang \& Graham, 2008). A distinct conventional perpetrator class might underestimate the psychosocial strain of cyberbullying perpetrators or misattribute indications of such strain to their perpetrator status instead of to their (albeit low-level) victimization experiences. Ignoring these experiences under the conventional classification approach may ignore important indications for theoretical assumptions regarding the etiology of cyberbullying 
perpetration. In this line, it is noteworthy that in the perpetrator class the probabilities are much higher to perpetrate and experience frequent acts than in the bully-victim class, which predominantly shows medium probabilities of verbal (e.g., insults, speak ill) and relational (e.g., exclusion) acts. This has important practical implications.

\section{Classification of Traditional Bullying Involvement and Comparison with Cyberbullying}

\section{Classes}

For traditional bullying, the LCA identified four classes. A five-class solution would also have been fitting, but was rejected because of very low proportions and interpretation difficulties. Based on statistical indicators and theoretical deliberations the simpler four-class model was favored. The four classes identified were: noninvolved, mild bully-victims, bullyvictims, and mainly perpetrators. There was a lack of a clear victim class and the two bullyvictim classes seem a mixture of behavior-oriented and severity-oriented classes such as the classes found by Wang and colleagues (2010) and Nylund and colleagues (2007).

A comparison of the results of the LCA for cyberbullying and traditional bullying must be met with caution due to methodological differences such as the different number of items as well as differences in their content. Nonetheless, a prominent difference is the number of extracted classes. More important differences, however, concern the interpretation of the classes as well as the proportions. Perpetrators in cyberbullying are less common (about 4\%), but commonly report victimization experiences at least to a low-frequency extent, while there are three times more perpetrators in traditional bullying who report low probabilities of being victimized themselves. The mild bully-victim class in traditional bullying and the bully-victim class in cyberbullying are to some extent comparable, especially regarding the types of behaviors reported (verbal and excluding behaviors); with proportions of $33.5 \%$ and $26.1 \%$, respectively, they are the most common classes of involved individuals. The remaining results of the LCA, however, indicate structural differences and lead to the 
assumption that there might also be differences in motivation for the behaviors (e.g., retaliation in cyberbullying).

\section{Comparison with Conventional Classification}

The cross-tabulations of class memberships according to the two different approaches (LCA vs. conventional cutoff score classification) show a good detection of noninvolved students. None of the conventional uninvolved individuals was classified as involved by LCA. Conversely, partly high proportions of involved students according to the conventional approach were classified as noninvolved by LCA. This might be due to the relatively low threshold we used for the conventional approach. However, both approaches were comparable as the LCA also included a low-frequency response category. Classification as bully-victims showed high consistency across both approaches and the majority of those classified as perpetrators by LCA belonged to the conventional bully-victim class. Nevertheless, compared to conventional classification, LCA produced lower rates of involvement, indicating that conventional classification overestimates involvement (only $57 \%$ of noninvolved in LCA were also noninvolved according to conventional classification). The conventional approach is too sensitive regarding single items, especially in the way it was used in this study insofar that single items lead to a classification as involved, while for LCA this was insufficient.

The decision about which approach to use in future studies needs to be informed by sound theoretical assumptions and solid arguments, bearing in mind the respective research objectives. In light of the present results, it does not seem advisable to hold on to classes neglecting that, for example, perpetrators also experience (infrequent) victimization.

\section{Association of Class Membership with Covariates}

Analyses of demographic covariates showed gender, age, and country differences. In line with the findings of Patchin and Hinduja (2012), girls were more likely to belong to the 
bully-victim class of cyberbullying. Looking at the predominant behaviors in this class it is not surprising, as members are mainly victimized through and perpetrate by saying nasty things to the victim's face or behind the victim's back as well as excluding the victim. These behaviors have previously been proposed (Crick \& Grotpeter, 1995)—but in part already disproved (Scheithauer, Haag, Mahlke, \& Ittel, 2008)— to be typical female forms of aggression. Also consistent with Patchin and Hinduja (2012), boys were perpetrators more often. This replicates often reported gender differences and numerous theories that boys are generally more aggressive. However, the result could possibly also be ascribed to gender stereotypes or social desirability where boys simply report less victimization, because it does not fit into their gender identity; for example, in a study on the impact of cyberbullying boys more often claimed to not be affected by it (Ortega et al., 2009).

Age differences indicate greater cyberbullying involvement with increasing age. This is also in line with research reported earlier. Reasons might be improved media-related skills, higher probabilities of being equipped with the respective technology, but also less parental monitoring of media-related activities with increasing age.

Membership in one of the two involvement groups of cyberbullying is also associated with the country where the respective data were collected. Greece and Poland had the highest odds for involvement as a perpetrator while Italy showed the highest and Germany the lowest odds for an individual being a bully-victim. The reasons for these differences can be manifold. The EU Kids Online study (Livingstone, Haddon, Görzig, \& Ólafsson, 2011), for example, showed that adolescents in Poland have Internet access in their own bedroom at home considerably more often than in Germany. Descriptive analyses of the present data also showed that adolescents from Poland report the highest daily Internet use and German adolescents report the lowest. Another possibility is an age x country interaction, as the mean age of participants differed among the participating countries and age could function as a 
confounding variable in the present country differences.

\section{Limitations and Outlook}

As mentioned before, the present study is the first to analyze classes of cyberbullying using LCA and no knowledge existed in this field to build on; it was necessary to draw from traditional bullying and peer harassment research where studies using LCA are also still scarce and even fewer include both victimization and perpetration.

As cross-national comparisons are always difficult, one limitation is the different mean ages of the various European samples. Also, the different sample sizes might have influenced the results through different weights. In the future, latent classes should be compared across countries in the way we have compared the results of LCA between cyberand traditional bullying. Possibly, the class structure differs across countries, although the satisfactorily fitting model in the present study suggests otherwise. We were also not able to include country $\mathrm{x}$ media use interactions as a statistical term in the present study due to parameter constraints.

Some limitations pertain to the general method of LCA. For example, it is noteworthy that LCA classified some individuals as bully-victims who were not identified as such by the more sensitive conventional approach. Theoretically, any individual with a positive answer on both victimization and perpetration would automatically have been classified as a conventional bully-victim. This phenomenon can be ascribed to the statistical specificities of the LCA method, which is based on probability values and not absolute scores. Further, if a victim's profile on the victimization items is similar to a bully-victim's, he or she will be assigned to the respective class. Interpretation of this is difficult and needs further investigation. For example, this might be an indicator of social desirability effects on the perpetration scale. Also, this might indicate whether this individual has been or will become a perpetrator later on. Allocation to a specific class based on probabilities might then possibly 
have predictive value for cyberbullying careers. Another interpretation might be that this effect can be solely attributed to statistical reasons. Moreover, classes that emerge in LCA might be dependent on the specific items and the number of items so that extracted classes could differ between samples. For adequate representations of the characteristics of involved individuals it is still advisable to use this method of post-hoc classification (Giang \& Graham, 2008) rather than possibly misclassifying individuals.

We have not reported on the discriminatory power of the single items here. However, these analyses might be helpful in reducing the length and optimizing the discriminatory power of existing assessment instruments. Preliminary views of these data suggest some items were irrelevant for discriminating between the extracted classes. Removing such items from future analyses might enable the inclusion of further covariates by raising the degrees of freedom and loosening the parameter constraints.

Also, the present classes should now be examined regarding meaningful adjustment problems and other outcomes. Longitudinal data should be collected to assess the stability of the extracted classes over time and developmental stages.

Nonetheless, the present study is the first of its kind to analyze the specific structure of the cyberbullying concept. It used a statistical method based on answer patterns and probabilities rather than predefined cutoff scores. We were able to show that traditional and cyber bullying differed in their structure and that the conventional classification approach using cutoff scores overestimates the prevalence of cyberbullying. Our study provides first insights into the profiles of involvement groups based on a large sample with students from six different European countries that can inform future intervention and prevention efforts.

\section{Competing Interests}

The authors report no known competing interests.

\section{Funding}


This research was supported by a research grant from the DAPHNE III program to combat violence against children, young persons and women of the European Commission (Action Number: JLS/2008/DAP3/AG/1211-30-CE-0311025/00-69; project title "Cyberbullying in Adolescence: Investigation and Intervention in Six European Countries" granted to the University of Bologna, Italy). The views expressed in this article are ours and do not represent the granting agency.

\section{Acknowledgements}

The authors thank Martin Schultze for his help in preparing the figures. 


\section{References}

Bradshaw, C. P., Waasdorp, T. E., \& O’Brennan, L. M. (2013). A latent class approach to examining forms of peer victimization. Journal of Educational Psychology, 105, 839849. doi:10.1037/a0032091

Brighi, A., Ortega, R., Pyzalski, J., Scheithauer, H., Smith, P. K., Tsormpatzoudis, H., et al. (2012). European Cyberbullying Intervention Project Questionnaire-ECIPQ. Unpublished questionnaire.

Crick, N. R., \& Grotpeter, J. K. (1995). Relational aggression, gender and socialpsychological adjustment. Child Development, 66, 710-722. doi:10.1111/j.14678624.1995.tb00900.x

Genta, M. L., Smith, P. K., Ortega, R., Brighi, A., Guarini, A., Thompson. F., et al. (2012). Comparative aspects of cyberbullying in Italy, England, and Spain: Findings from a DAPHNE project. In Q. Li, D. Cross, \& P. K. Smith (Eds.), Cyberbullying in the global playground: Research from international perspectives (pp. 13-32). Chichester, UK: Wiley-Blackwell.

Giang, M. T., \& Graham, S. (2008). Using latent class analysis to identify aggressors and victims of peer harassment. Aggressive Behavior, 34, 203-213. doi:10.1002/ab.20233

Görzig, A., \& Ólafsson, K. (2013). What makes a bully a cyberbully? Unravelling the characteristics of cyberbullies across twenty-five European countries. Journal of Children and Media, 7, 9-27. doi:10.1080/17482798.2012.739756

Kowalski, R. M., Guimetti, G. W., Schroeder, A. N., \& Lattanner, M. R. (2014). Bullying in the digital age: A critical review and meta-analysis of cyberbullying research among youth. Psychological Bulletin, 140, 1073-1137. doi:10.1037/a0035618

Langos, C. (2012). Cyberbullying: The challenge to define. CyberPsychology, Behavior \& Social Networking, 15, 285-289. doi:10.1089/cyber.2011.0588 
Law, D. M., Shapka, J. D., Hymel, S., Olson, B. F., \& Waterhouse, T. (2012). The changing face of bullying: An empirical comparison between traditional and internet bullying and victimization. Computers in Human Behavior, 28, 226-232. doi:10.1016/j.chb.2011.09.004

Li, Q., Smith, P. K., \& Cross, D. (2012). Research into cyberbullying: Context. In Q. Li, D. Cross, \& P. K. Smith (Eds.), Cyberbullying in the global playground. Research from international perspectives (pp. 3-12). Chichester, UK: Wiley-Blackwell.

Livingstone, S., Haddon, L., Görzig, A., \& Ólafsson, K. (2011). EU Kids Online final report. Retrieved from www.1se.ac.uk/media@1se/research/EUKidsOnline/EU\%20Kids\%20II\%20\%28200911\%29/EUKidsOnlineIIReports/Final\%20report.pdf

Lobe, B., Livingstone, S., Ólafsson, K., \& Vodeb, H. (2011). Cross-national comparison of risks and safety on the internet: Initial analysis from the EU Kids Online survey of European children. London, UK: EU Kids Online Network.

Lovegrove, P. J., Henry, K. L., \& Slater, M. D. (2012). Examination of the predictors of latent class typologies of bullying involvement among middle school students. Journal of School Violence, 11, 75-93. doi:10.1080/15388220.2011.631447

Menesini, E., Nocentini, A., \& Calussi, P. (2011). The measurement of cyberbullying: Dimensional structure and relative item severity and discrimination. CyberPsychology, Behavior \& Social Networking, 14, 267-274. doi:10.1089/cyber.2010.0002

Muthén, L. K., \& Muthén, B. O. (1998-2012). Mplus user's guide (7th ed.). Los Angeles, CA: Muthén \& Muthén.

Nylund, K., Bellmore, A., Nishina, A., \& Graham, S. (2007). Subtypes, severity, and structural stability of peer victimization: What does latent class analysis say? Child 
Development, 78, 1706-1722. doi:10.1111/j.1467-8624.2007.01097.x

Olweus, D. (1993). Bullying at school: What we know and what we can do. Oxford, UK: Blackwell.

Olweus, D. (2012). Cyberbullying: An overrated phenomenon? European Journal of Developmental Psychology, 9, 520-538. doi:10.1080/17405629.2012.682358

Ortega, R., Elipe, P., Mora-Merchán, J. A., Calmaestra, J., \& Vega, E. (2009). The emotional impact on victims of traditional bullying and cyberbullying: A study of Spanish adolescents. Zeitschrift für Psychologie / Journal of Psychology, 217, 197-204. doi:10.1027/0044-3409.217.4.197

Ortega, R., Elipe, P., Mora-Merchán, J. A., Genta, M. L., Brighi, A., Guarini, A., et al. (2012). The emotional impact of bullying and cyberbullying on victims: A European cross-national study. Aggressive Behavior, 38, 342-356. doi:10.1002/ab.21440

Patchin, J. W., \& Hinduja, S. (2012). Cyberbullying: An update and synthesis of the research. In J. W. Patchin \& S. Hinduja (Eds.), Cyberbullying prevention and response: Expert perspectives (pp. 13-35). New York, NY: Routledge.

Perren, S., Dooley, J., Shaw, T., \& Cross, D. (2010). Bullying in school and cyberspace: Associations with depressive symptoms in Swiss and Australian adolescents. Child and Adolescent Psychiatry and Mental Health, 4(28). doi:10.1186/1753-2000-4-28

Scheithauer, H., Haag, N., Mahlke, J., \& Ittel, A. (2008). Gender and age differences in the development of relational/indirect aggression: First results of a meta-analysis. European Journal of Developmental Science, 2, 176-189. doi:10.3233/DEV-200821211

Smith, P. K. (2010). Cyberbullying: The European perspective. In J. A. Mora-Merchán \& T. Jäger (Eds.), Cyberbullying: A cross-national comparison (pp. 7-19). Landau, Germany: Verlag Empirische Pädagogik. 
Suler, J. (2004). The online disinhibition effect. CyberPsychology \& Behavior, 7, 321-326. doi:10.1089/1094931041291295

Suzuki, K., Asaga, R., Sourander, A., Hoven, C. W., \& Mandell, D. (2012). Cyberbullying and adolescent mental health. International Journal of Adolescent Medicine and Health, 24(1), 27-35. doi:10.1515/IJAMH.2012.005

Tokunaga, R. S. (2010). Following you home from school: A critical review and synthesis of research on cyberbullying victimization. Computers in Human Behavior, 26, 277287. doi:10.1016/j.chb.2009.11.014

Vermunt, J. K. (2010). Latent class modeling with covariates: Two improved three-step approaches. Political Analysis, 18, 450-469. doi:10.1093/pan/mpq025

Waasdorp, T. E., \& Bradshaw, C. P. (2011). Examining student responses to frequent bullying: A latent class approach. Journal of Educational Psychology, 103, 336-352. doi:10.1037/a0022747

Wang, J., Iannotti, R. J., Luk, J. W., \& Nansel, T. R. (2010). Co-occurrence of victimization from five subtypes of bullying: Physical, verbal, social exclusion, spreading rumors, and cyber. Journal of Pediatric Psychology, 35, 1103-1112. doi:10.1093/jpepsy/jsq048

Williford, A. P., Brisson, D., Bender, K. A., Jenson, J. M., \& Forrest-Bank, S. (2011). Patterns of aggressive behavior and peer victimization from childhood to early adolescence: A latent class analysis. Journal of Youth and Adolescence, 40, 644-655. doi:10.1007/s10964-010-9583-9

Wolak, J., Mitchell, K., \& Finkelhor, D. (2007). Does online harassment constitute bullying? An exploration of online harassment by known peers and online-only contacts. Journal of Adolescent Health, 41, S51-S58. doi:10.1016/j.jadohealth.2007.08.019 
Running head: Classification Approaches for Cyberbullying

Table 1

Sample Characteristics for Each Country

\begin{tabular}{lrcrc}
\hline Country & $N$ of schools & $N$ of pupils & Age Mean & $\%$ Female \\
\hline Poland & 8 & 1,002 & 14.1 & 49.3 \\
Spain & 3 & 892 & 13.8 & 45.7 \\
Italy & 15 & 1,697 & 16.4 & 54.5 \\
UK & 5 & 771 & 15.1 & 41.8 \\
Germany & 5 & 895 & 13.5 & 51.4 \\
Greece & 13 & 1003 & 14.9 & 50.8 \\
\hline Total & 49 & 6,260 & 14.8 & 49.9 \\
\hline
\end{tabular}


Table 2

Behaviors Assessed for the Measurement Cyberbullying and Traditional Bullying (and Their Respective Number for Later Reference in the Diagrams)

Cybervictimization / Cyberbullying Traditional victimization / Traditional bullying

Say nasty things or call someone names using Hit, kick or push (Physical) texts or online messages (Insults)

Say nasty things about someone to others either online or through text messages

Say nasty things or call someone names (Insults) (Speak ill)

Threaten through texts or online messages (Threats)

Hack into someone's account and steal personal information (Data theft)

Say nasty things about someone to others (Speak ill)

Threaten (Threats)

Hack into someone's account and pretend to be Steal or damage others' belongings (Theft / that person (Impersonation)

Damage)

Create a fake account pretending to be that person (Fake account)

Post personal information of others online Spread rumors about others (Rumors) (Exposure secrets)

Post embarrassing videos or pictures of others online (Exposure pictures)

Alter pictures or videos of others they had posted online (Photoshop)

Exclude or ignore others in a social networking site or internet chat room (Exclusion)

Spread rumors about others on the Internet (Rumors)

Note. Key terms in parentheses indicates reference key term in Figure 1. 
Table 3

Latent Class Analyses Fit Indices for Cyber- and Traditional Bullying $(N=6,260)$

\begin{tabular}{lcccc}
\hline No. of classes & AIC & BIC & aBIC & Log-likelihood \\
\hline & & Cyberbullying & \\
2 classes & 80134.368 & 80734.400 & 80451.582 & -39978.184 \\
$\mathbf{3}$ classes & $\mathbf{7 6 8 6 3 . 8 5 9}$ & $\mathbf{7 7 7 6 7 . 3 8 5}$ & $\mathbf{7 7 3 4 1 . 5 6 8}$ & $\mathbf{- 3 8 2 9 7 . 9 2 9}$ \\
4 classes & 75904.588 & 77111.395 & 76542.580 & -37773.294 \\
5 classes & 75231.573 & 76741.767 & 76029.954 & -37391.787 \\
\hline & & Traditional bullying & \\
2 classes & 108130.457 & 108514.748 & 108333.617 & -54008.229 \\
3 classes & 104879.994 & 105459.801 & 105186.515 & -52353.997 \\
$\mathbf{4}$ classes & $\mathbf{1 0 3 0 0 0 . 9 3 6}$ & $\mathbf{1 0 3 7 7 6 . 3 5 0}$ & $\mathbf{1 0 3 4 1 0 . 9 1 1}$ & $\mathbf{- 5 1 3 8 5 . 4 6 8}$ \\
5 classes & 101873.754 & 102844.592 & 102386.999 & -50792.877 \\
\hline
\end{tabular}

Note. AIC $=$ Akaike Information Criteria $; \mathrm{BIC}=$ Bayesian Information Criteria; $\mathrm{aBIC}=$ Adjusted BIC. Bolded classes indicate the best-fitting model. 
Table 4

Comparison of the LCA Approach to the Conventional Classification Method for

Cyberbullying $(N=6,260)$

Conventional classification

method $(\%)$

Latent classes for cyberbullying (\%)

\begin{tabular}{ccc}
\hline Noninvolved & Bully-victims & $\begin{array}{c}\text { Perpetrators with mild } \\
\text { victimization }\end{array}$ \\
\hline 57.4 & 0.0 & 0.0 \\
28.1 & 26.4 & 3.8 \\
5.8 & 67.2 & 90.2 \\
8.7 & 6.4 & 6.0 \\
\hline
\end{tabular}

Latent classes for

cyberbullying $(\%)$

Conventional classification method (\%)

\begin{tabular}{lcccc}
\hline & Noninvolved & Victims & Bully-victims & Bullies \\
Noninvolved & 100.0 & 75.0 & 17.3 & 77.6 \\
Bully-victims & 0.0 & 24.4 & 68.8 & 19.8 \\
Perpetrators & 0.0 & 0.5 & 13.9 & 2.8 \\
\hline
\end{tabular}


Running head: Classification Approaches for Cyberbullying

\section{Figure Caption}

Figures $1 a-c$. Item probability profile plots for each class of cyberbullying. Plots contain three response categories: never, once or twice, and more than 2-3 times a month (corresponding to dark to light shading) $(N=6,260)$. 